\title{
ВЗАИМОСВЯЗЬ ИЗМЕНЕНИЙ ПОКАЗАТЕЛЕЙ ФУНКЦИОНАЛЬНОГО СОСТОЯНИЯ И ДОППЛЕР-ЭХОКАРДИОГРАФИИ У БОЛЬНЫХ ОСТРЫМ ИНФАРКТОМ МИОКАРДА НА ФОНЕ РЕАБИЛИТАЦИИ
}

\author{
С Михин В.П. ${ }^{1}$, Коробова В.Н. ${ }^{1}$, Харченко А.В. ${ }^{1}$, Громнацкий Н.И. ${ }^{1}$, Выгодин В.А. ${ }^{2}$ \\ ${ }^{1}$ Курский государственный медицинский университет (КГМУ) \\ Россия, 305041, Курская область, г. Курск, ул. К. Маркса, д. 3 \\ ${ }^{2}$ Национальный медицинский исследовательский центр профилактической медицины
}

(НМИЦ ПМ)

Россия, 101990, г. Москва, Петроверигский пер., д. 10

\begin{abstract}
Целью исследования явилась оценка интегральных показателей функционального состояния (ФС) организма, параметров центральной и внутрисердечной гемодинамики больных острым инфарктом миокарда в процессе госпитальной и постгоспитальной реабилитации, изучение взаимосвязи между полученными данными.

Материалы и методы. Открытое рандомизированное исследование включало 111 человек (76 мужчин и 35 женщин) в возрасте от 40 до 65 лет $(57,2 \pm 0,6)$. Исследование ФС организма осуществлялось при помощи аппаратно-программного комплекса «Динамика-Омега-М» на 3-и, 8-е, 14-е сут и к 6-му и 12-му мес. Допплерэхокардиография проводилась на 3-и и 14-е сут пребывания в стационаре, через 6 и 12 мес после выписки.

Полученные результаты позволили установить снижение значений интегральных показателей ФС на этапах госпитальной и постгоспитальной реабилитации, наличие гипертрофии миокарда, нарушение диастолической функции сердца и локальной сократимости. На фоне реабилитации была установлена положительная динамика как параметров ФС, так и показателей, характеризующих внутрисердечную гемодинамику. Установлены значимые корреляционные связи значений параметров ФС с эхокардиографическими показателями и их динамикой во время исследования.
\end{abstract}

Заключение. В работе обоснована целесообразность использования интегральных параметров ФС для комплексной оценки статуса больных острым инфарктом миокарда и в качестве прогностических критериев эффективности реабилитации.

Ключевые слова: острый инфаркт миокарда; функциональное состояние; внутрисердечная гемодинамика; центральная гемодинамика; реабилитация.

Михин Вадим Петрович - д-р. мед. наук, профессор, зав. кафедрой внутренних болезней № 2, КГмУ, г. Курск. ORCID iD: 0000-0002-5398-9727. E-mail: mikhinvp@yandex.ru

Коробова Виктория Николаевна - ассистент кафедры патофизиологии, КГМУ, г. Курск. ORCID iD: 0000-0002-2737-3435. E-mail: viktoria.korobova@mail.ru (автор, ответственный за переписку)

Харченко Александр Владимирович - канд. мед. наук, доцент кафедры внутренних болезней № 2, КГМУ, г. Курск. ORCID iD: 0000-0001-8636-3096. E-mail: hav46@mail.ru

Громнацкий Николай Ильич - д-р. мед. наук, профессор, профессор кафедры внутренних болезней № 2, КГмУ, г. Курск. E-mail: gromnackini@yandex.ru

Выгодин Владимир Анатольевич - ст. науч. сотрудник лаборатории медицинской биостатистики, НМИЦ ПМ, г. Москва. E-mail: vladimirvygodin@mail.ru

Острый инфаркт миокарда (ОИМ) продолжает оставаться одной из ведущих причин инвалидизации и смертности в структуре ишемической болезни сердца [15]. Несмотря на появление новых методов и медикаментозных препаратов для лечения больных ОИМ, кардиореабилитация этой категории больных является одной из приоритетных задач кардиологии [3]. Возникающие в результате нарушений коронарного кровотока зоны некроза в миокарде ассоциированы с нарушениями внутрисердечной и центральной гемодинамики, которые регистрируются при допплер-эхокардиографии (допплер-Эхо-КГ). Допплер-Эхо-КГ позволяет оценить степень нарушения систолодиастолической функции сердца, что используется для определения эффективности реабилитации $[1,2,4]$. Тем не менее на клинический статус больных ОИМ влияют не только гемодинамические нарушения, но и целый ряд дополнительных факторов, в частности дисбаланс со стороны вегетативной и центральной нервной системы, психоэмоциональное состояние, уровень адаптации организма $[6,8,14,17]$. Одним из методов комплексного анализа функциональных расстройств больных ОИМ признан математический анализ кардиоритма с использованием линейных и нелинейных способов обработки биоритмологического процесса [10, 11]. В настоящее время разработаны аппаратнопрограммные комплексы (АПК), позволяющие на основе линейных и нелинейных методик обработки кардиосигнала получать интегральные показатели адаптации сердечно-сосудистой системы, вегетативной и центральной регуляции, психоэмоционального состояния. В этой связи 
представляется перспективным использование указанного АПК для оценки функционального состояния (ФС) больных ОИМ, поскольку нестабильность их состояния может быть ассоциирована не столько с глубиной поражения миокарда, сколько с психоэмоциональным компонентом. В настоящее время особенности параметров ФС у больных с острой коронарной патологией изучены недостаточно, хотя при других состояниях (дерматозы, хронический болевой синдром) указанные параметры изучались [7, 9]. Остаются совсем неизученными взаимосвязи параметров ФС с показателями центральной и внутрисердечной гемодинамики, оцененные методом допплер-Эхо-КГ. В этой связи поиск взаимосвязи и характера изменений показателей центральной, внутрисердечной гемодинамики и параметров ФС представляется важным для разносторонней оценки эффективности лечения и реабилитации больных ОИМ и выявления значимых маркеров адекватности проводимой терапии. Хотя патофизиологическая связь указанных показателей представляется очевидной.

Цель работы: оценить интегральные показатели ФС организма и их взаимосвязь с параметрами центральной и внутрисердечной гемодинамики у больных ОИМ в процессе госпитальной и постгоспитальной реабилитации.

\section{МАТЕРИАЛЫ И МЕТОДЫ ИССЛЕДОВАНИЯ}

Открытое рандомизированное исследование включало 111 человек (76 мужчин и 35 женщин) в возрасте от 40 до 65 лет $(57,2 \pm 0,6)$. Критериями включения были возраст, наличие ОИМ, регулярный синусовый ритм на этапах исследования, информированное согласие. Критерии исключения: кардиогенный шок, операции в ближайшие 6 месяцев, отказ от участия в исследовании.

В первые два дня госпитализации у 9,1\% больных был зарегистрирован отек легких $(\mathrm{n}=9)$, у $1,8 \%$ - сердечная астма $(\mathrm{n}=2)$, у 1,8\% - гипертонический криз $(\mathrm{n}=2)$. Сопутствующая патология: у 21,6\% больных - постинфарктный кардиосклероз $(\mathrm{n}=24), \mathrm{y}$ 0,1\% - бронхиальная астма $(\mathrm{n}=1), \mathrm{y}$ $2,7 \%$ - хроническая обструктивная болезнь легких $(\mathrm{n}=3)$, у 5,4\% - энцефалопатия (n=6), у 10,8\% последствия острой недостаточности мозгового кровообращения $(\mathrm{n}=12)$, у 11,2\% - цереброваскулярная болезнь $(\mathrm{n}=13)$, у 5,4\% - язвенная болезнь желудка $(\mathrm{n}=6)$, у 4,5\% - язвенная болезнь двенадцатиперстной кишки $(\mathrm{n}=5), \mathrm{y} 4,5 \%$ - хроническая болезнь почек $(\mathrm{n}=5)$, у $27 \%$ - сахарный диабет 2 типа $(\mathrm{n}=30)$.
Медикаментозная терапия соответствовала стандартам и включала: наркотические и ненаркотические анальгетики, антиагреганты и гепарин, бета-блокаторы, ингибиторы АПФ или сартаны, статины, нитраты и петлевые диуретики при необходимости $[15,16]$. Тромболитическая терапия была проведена у трех больных $(2,7 \%)$ с положительным эффектом, выполнение ангиопластики было невозможно в связи с отсутствием необходимого оборудования или наличием противопоказаний.

ФС организма пациентов оценивалось с использованием АПК «Динамика-Омега-М» (OOО «Торговый дом "Динамика"», г. Москва; регистрационное удостоверение № ФСР 2010/09117 от 01.11.2010 г. Федеральной службы по надзору в сфере Здравоохранения и социального развития) на 3-е, 8-е и 14-е сутки пребывания в стационаре и через 6 и 12 мес. Анализировались следующие интегральные показатели ФС организма: A - показатель адаптации сердечнососудистой системы, В - показатель вегетативной регуляции, С - показатель центральной регуляции, D - показатель психоэмоционального состояния, H - показатель здоровья [5, 12]. Норма значений указанных показателей ФС для здоровых лиц 60-100\%, референсные значения для кардиологических больных в настоящее время не разработаны [11]. Допплер-Эхо-КГ проводилась на 3-и и 14-е сутки пребывания в стационаре, а также через 6 и 12 мес на ультразвуковой системе Esaote Megas CVX (Италия) с использованием метода допплер-эхокардиографии при помощи датчика Biosound (Esaote) 3550 АА-С 7,5/10,0 MHz с оценкой следующих показателей: конечно-диастолический размер (КДР) левого желудочка (ЛЖ), конечносистолический размер (КСР) ЛЖ, толщина межжелудочковой перегородки (МЖП) в систолу и в диастолу, толщина задней стенки ЛЖ (ЗС ЛЖ), фракция выброса (ФВ), максимальная скорость раннего пика диастолического наполнения (пик Е), максимальная скорость трансмитрального кровотока во время систолы левого предсердия (пик А), отношение максимальных скоростей раннего и позднего наполнения (E/A), время изоволюмического расслабления (IVRT) и сокращения (IVCT) ЛЖ, время замедления раннего диастолического наполнения (DTE), индекс локальной сократимости (ИЛС) миокарда [4]. Динамические изменения значений показателей допплер-Эхо-КГ оценивали следующие временные промежутки: от 3-х сут к 14-м сут, 6-му и 12му мес, от 14-х сут к 6-му и 12-му мес.

Корреляционный анализ проводился между величинами интегральных показателей ФС организма на каждом из этапов исследования, значениями эхокардиографических показателей 
и их динамикой на фоне реабилитации. Коэффициенты корреляции рассчитывали с использованием методов линейной корреляции по Пирсону (r), ранговой корреляции по Спирмену (R) и ранговой корреляции по Кендаллу (Tau-b), статистическая значимость которых оценивалась системой SAS (Statistical Analysis System, SAS Institute, Cary, NC, USA) по соответствующим формулам. Для оценки силы связи использовали следующую градацию: $<0,25$ - слабая, 0,25-0,5 - средняя, 0,5-0,75 - сильная, 0,75-1 очень сильная. Уровень значимости $\mathrm{p}$ был определен менее 0,05 .

\section{РЕЗУЛЬТАТЫ ИССЛЕДОВАНИЯ И ИХ ОБСУЖДЕНИЕ}

При оценке интегральных показателей ФС организма больных ОИМ было установлено, что на этапах госпитальной и постгоспитальной реабилитации значения показателей $\mathrm{A}, \mathrm{B}, \mathrm{C}, \mathrm{D}$ и $\mathrm{H}$ были ниже нормы (табл. 1).

Обращало внимание, что в процессе реабилитации величина показателя адаптации сердечно-сосудистой системы (А), центральной регуляции (С), психоэмоционального состояния (D) и интегрального показателя здоровья (H) увеличивалась от этапов госпитальной реабилитации к этапам постгоспитальной реабилитации. В то время как значение показателя вегетативной регуляции (В) снижалось от госпитальных этапов к 6-му и 12-му мес. Указанные изменения показателя В у больных ОИМ, возможно, были связаны с использованием в лечении бетаблокаторов, механизм действия которых направлен на подавление адренергических симпатических влияний вегетативной нервной системы. Тем не менее среднее значение всех показателей $\Phi С$ на этапах госпитальной и постгоспитальной реабилитации было ниже $37 \%$, что значительно ниже установленной нормы для здоровых лиц, при этом исследования по оценке интегральных показателей ФС кардиологических больных с помощью АПК «ДинамикаОмега-М» в литературе не найдены. В литературе были найдены данные больных, проходящих лечение по поводу атопического дерматита: минимальное значение было зарегистрировано в отношении значений показателей $\mathrm{B}$ и $\mathrm{C}$ (42 и $43 \%$ соответственно), что позволило сделать вывод о превалирующем значении в патогенезе атопического дерматита вегетативного и центрального уровней регуляции. Таким образом, столь значимое отклонение от нормы всех интегральных показателей $\Phi С$ на этапах госпитальной и постгоспитальной реабилитации свидетельствовало о наличие серьезных нарушений функционирования организма больных ОИМ на всех уровнях регуляции. Положительная динамика величины показателей $\mathrm{A}, \mathrm{C}, \mathrm{D}$ и $\mathrm{H}$ в ходе исследования подтверждала эффективность реабилитационного периода.

Как видно из таблицы 2, структурные параметры сердца показали увеличение ЗС ЛЖ и МЖП, что свидетельствует о наличие гипертрофии миокарда у больных ОИМ. На этапах госпитальной и постгоспитальной реабилитации было установлено нарушение диастолической функции ЛЖ, поскольку регистрировалось увеличение значений показателя Peak А и снижение значений Peak E и E/A. КДР и КСР ЛЖ не выходили за пределы нормы, тем не менее анализ динамических изменений установил увеличение значений КДР ЛЖ и уменьшение

Таблица 1 Table 1

Функциональное состояние больных ОИМ

на этапах госпитальной и постгоспитальной реабилитации $(\mathrm{M} \pm \mathrm{m})$

The functional state of patients with AMI at the stages of hospital and post-hospital rehabilitation $(\mathrm{M} \pm \mathrm{m})$

\begin{tabular}{|c|c|c|c|c|c|}
\hline \multirow{2}{*}{$\begin{array}{c}\text { Параметры } \\
\text { FS parameters }\end{array}$} & \multicolumn{5}{|c|}{$\begin{array}{c}\text { Сроки наблюдения } \\
\text { Terms of observation }\end{array}$} \\
\cline { 2 - 6 } & $\begin{array}{c}\text { 3-и сутки } \\
3^{\text {rd }} \text { day }\end{array}$ & $\begin{array}{c}\text { 8-е сутки } \\
8^{\text {th }} \text { day }\end{array}$ & $\begin{array}{c}\text { 14-е сутки } \\
14^{\text {th }} \text { day }\end{array}$ & $\begin{array}{c}\text { 6-й месяц } \\
6^{\text {th }} \text { month }\end{array}$ & $\begin{array}{c}12 \text {-й месяц } \\
12^{\text {th }} \text { month }\end{array}$ \\
\hline $\mathrm{A}$ & $19.1 \pm 1.7^{* 6,12}$ & $18.3 \pm 1.8^{* 6,12}$ & $19.1 \pm 1.7^{6,12}$ & $23.9 \pm 2.1$ & $26.2 \pm 2.2$ \\
\hline $\mathrm{B}$ & $33.7 \pm 2.4^{* 6,12}$ & $34.3 \pm 2.5^{* 6,12}$ & $36.1 \pm 2.4^{* 6,12}$ & $23.9 \pm 2.1$ & $26.2 \pm 2.1$ \\
\hline $\mathrm{C}$ & $20.8 \pm 1.7^{* 6,12}$ & $21.8 \pm 1.9^{* 6,12}$ & $21.7 \pm 1.8^{* 6,12}$ & $29.2 \pm 2.1$ & $32.2 \pm 2.2$ \\
\hline $\mathrm{D}$ & $22.4 \pm 1.7^{* 6,12}$ & $23.1 \pm 1.9^{* 6,12}$ & $24.1 \pm 1.9^{* 6,12}$ & $31.7 \pm 2$ & $35.8 \pm 2.1$ \\
\hline $\mathrm{H}$ & $24 \pm 1.8^{* 6,12}$ & $24.4 \pm 1.9^{* 6,12}$ & $25.2 \pm 1.8^{* 6,12}$ & $31.2 \pm 2.1^{* 12}$ & $34.4 \pm 2.2$ \\
\hline
\end{tabular}

Примечание: * - достоверные динамические изменения $(\mathrm{p}<0,05)$ к сроку, указанному рядом цифрой: ${ }^{6}$ - 6-й мес, ${ }^{12}-12$-й мес.

Note: ${ }^{*}$ - reliable dynamic changes $(\mathrm{p}<0.05)$ by the date indicated by the next digit: ${ }^{6}-6^{\text {th }}$ month, ${ }^{12}-12^{\text {th }}$ month. FS - functional state, $\mathrm{A}$ is an indicator of the adaptation of the cardiovascular system, $\mathrm{B}$ is an indicator of vegetative regulation, $\mathrm{C}$ is an indicator of central regulation, $\mathrm{D}$ is an indicator of psycho-emotional state, $\mathrm{H}$ is an indicator of health. 
Результаты допплер-Эхо-КГ больных ОИМ

на этапах госпитальной и постгоспитальной реабилитации $(\mathrm{M} \pm \mathrm{m})$

Results of Doppler-Echo-CG in patients with AMI at the stages of hospital and post-hospital rehabilitation $(\mathrm{M} \pm \mathrm{m})$

\begin{tabular}{|c|c|c|c|c|}
\hline \multirow{2}{*}{$\begin{array}{c}\text { Параметры } \\
\text { Parameters }\end{array}$} & \multicolumn{4}{|c|}{$\begin{array}{l}\text { Сроки наблюдения } \\
\text { Terms of observation }\end{array}$} \\
\hline & $\begin{array}{c}\text { 3-и сутки } \\
3^{\text {rd }} \text { day }\end{array}$ & $\begin{array}{l}\text { 14-е сутки } \\
14^{\text {th }} \text { day }\end{array}$ & $\begin{array}{l}\text { 6-й месяц } \\
6^{\text {th }} \text { month }\end{array}$ & $\begin{array}{l}\text { 12-й месяц } \\
12^{\text {th }} \text { month }\end{array}$ \\
\hline $\begin{array}{l}\text { КДР ЛЖ, мм } \\
\text { FDS LV, mm }\end{array}$ & $44.9 \pm 0.7^{* 6}$ & $45.0 \pm 0.6^{* 6,12}$ & $45.7 \pm 0.6$ & $45.7 \pm 0.5$ \\
\hline $\begin{array}{l}\text { КСР ЛЖ, мм } \\
\text { FSS LV, mm }\end{array}$ & $30.0 \pm 0.7^{* 6,12}$ & $29.5 \pm 0.7$ & $28.9 \pm 0.6$ & $28.8 \pm 0.6$ \\
\hline $\begin{array}{l}\text { МЖП, мм } \\
\text { IVS, mm }\end{array}$ & $14.6 \pm 0.2$ & $14.6 \pm 0.2$ & $14.8 \pm 0.2$ & $14.6 \pm 0.2$ \\
\hline $\begin{array}{l}\text { 3С ЛЖ, мм } \\
\text { PW LV, mm }\end{array}$ & $13.2 \pm 0.1^{* 12}$ & $13.1 \pm 0.1$ & $13.0 \pm 0.2$ & $12.9 \pm 0.2$ \\
\hline $\begin{array}{l}\Phi B, \% \\
\mathrm{EF}, \%\end{array}$ & $55.4 \pm 0.9^{*^{6,12}}$ & $55.9 \pm 0.9^{* 6,12}$ & $58.3 \pm 0.9$ & $59.1 \pm 0.9$ \\
\hline Peak E & $0.59 \pm 0.04$ & $0.62 \pm 0.02$ & $0.62 \pm 0.02$ & $0.62 \pm 0.02$ \\
\hline Peak A & $0.86 \pm 0.10$ & $0.79 \pm 0.04$ & $0.81 \pm 0.04$ & $0.79 \pm 0.04$ \\
\hline $\mathrm{E} / \mathrm{A}$ & $0.76 \pm 0.07$ & $0.88 \pm 0.05$ & $0.87 \pm 0.04$ & $0.90 \pm 0.05$ \\
\hline IVRT & $84.7 \pm 4.6$ & $87.4 \pm 2.4$ & $87.0 \pm 2.6$ & $85.4 \pm 2.3$ \\
\hline IVCT & $45.5 \pm 1.9$ & $46.3 \pm 1.4$ & $49.2 \pm 1.9$ & $48.1 \pm 1.2$ \\
\hline DT & $241.5 \pm 27.6$ & $254.0 \pm 9.5$ & $236.6 \pm 7.3$ & $236.9 \pm 7.1$ \\
\hline $\begin{array}{l}\text { ИЛС } \\
\text { ILMC }\end{array}$ & $1.28 \pm 0.03^{\star 14,6,12}$ & $1.25 \pm 0.03^{* 6,12}$ & $1.20 \pm 0.02^{\star 12}$ & $1.18 \pm 0.02$ \\
\hline
\end{tabular}

Примечание: * - достоверные динамические изменения $(\mathrm{p}<0,05)$ к сроку, указанному рядом цифрой:

14 - 14-е сут, ${ }^{6}$ - 6-й мес, ${ }^{12}-12$-й мес.

Note: ${ }^{*}$ - significant dynamic changes $(\mathrm{p}<0.05)$ by the date indicated by the next digit: ${ }^{14}-14^{\text {th }}$ day, ${ }^{6}-6^{\text {th }}$ month, ${ }^{12}-12^{\text {th }}$ month. FDS LV - final-diastolic size of the left ventricle, FSS LV - final-systolic size of the left ventricle, IVS - thickness of the interventricular septum, PW LV - thickness of the posterior wall of the left ventricle, EF - ejection fraction, ILMC - index of local myocardial contractility.

КСР ЛЖ от 3-х сут к постгоспитальным этапам. Увеличение ФВ от 3-х и 14-х сут к 6-му и 12-му месяцу, а также снижение ИЛС миокарда в отношении каждого последующего этапа исследования позволили подтвердить эффективность проводимой кардиореабилитации.

Таким образом, вышеуказанные изменения ряда показателей как ФС, так и допплер-Эхо-КГ, имеющие положительную динамику в процессе госпитальной и постгоспитальной реабилитации, соответствуют клиническим признакам благоприятного течения восстановительного периода у больных ОИМ [13]. Проведенный корреляционный анализ параметров ФС и показателей внутрисердечной гемодинамики позволил установить наличие сильных и средних связей между отдельными показателями ФС и Эхо-КГ (табл. 3).

Тесные прямые корреляционные взаимосвязи были установлены между динамикой КДР ЛЖ в период 3-х - 14-х сут и 3-х сут - 6-го мес и показателем А на 3-и сут $(0,29$ и 0,34 соответственно), а КДР ЛЖ в период 3-х сут - 12-го мес коррелировал с показателем В на 3-и сут $(0,34)$.
Изменение КСР ЛЖ от 3-х к 14-м сут взаимосвязано с величиной показателя А на $3-и$ сут $(0,26)$. Значение ФВ ЛЖ на 14-е сут и 6-й мес имело прямую связь средней силы $(0,32$ и 0,37 соответственно) со значением показателя $\mathrm{C}$ на 3 -и сут, в то время как ФВ ЛЖ на 12-й мес коррелировала с С на 8-е сут $(0,37)$. Динамика $Ф В$ ЛЖ от 3 -х сут к 14-м сут была взаимосвязана с показателем С на 3-и сут $(0,39)$, динамика ФВ ЛЖ от 3-х сут к 6-му мес - с А на 8-е сут $(0,39)$, динамика ФВ ЛЖ от 3-х сут к 12-му мес равнозначно коррелировала со значениями двух интегральных показателей А и Н на 3-и сут $(0,41)$. Также была установлена корреляционная взаимосвязь динамики ФВ ЛЖ от 14-х сут к 6-му с величиной параметров A, B, С на 6-й мес $(0,28)$, а к 12-му мес - со значениями показателя А на 3-и сут и 6-й мес, В и С на 6-й мес. Изменение IVCT от 3-х сут к 12-му мес имело сильную связь со значением А на 3 -и $(0,52)$ и 8 -е сут $(0,70)$. Динамика показателя Е/А от 3-х сут к 14-м сут и 6-му мес имела сильную взаимосвязь с показателем D на 3-и сут $(0,55$ и 0,58 соответственно). Обратная корреляционная связь была установлена между ИЛС 
Таблица 3

Table 3

Результаты корреляционного анализа показателей ФС и параметров допплер-Эхо-КГ у больных острым инфарктом миокарда (r)

The results of the correlation analysis of FS parameters and Doppler-Echo-CG parameters in patients with acute myocardial infarction (r)

\begin{tabular}{|c|c|c|c|c|c|}
\hline \multirow{2}{*}{$\begin{array}{l}\text { Параметры допплер-Эхо-КГ } \\
\text { Parameters of doppler Echo-CG }\end{array}$} & \multicolumn{5}{|c|}{$\begin{array}{c}\text { Интегральные параметры ФС } \\
\text { Integral parameters of the functional state }\end{array}$} \\
\hline & A & B & $\mathrm{C}$ & $\mathrm{D}$ & $\mathrm{H}$ \\
\hline \multicolumn{6}{|c|}{$\begin{array}{l}\text { 3-и сутки } \\
3^{\text {rd }} \text { day }\end{array}$} \\
\hline $\begin{array}{l}\Delta \mathrm{KДР}_{3-14} \\
\Delta \mathrm{FDS}_{3-14}\end{array}$ & 0.29 & $\begin{array}{l}\mathrm{c} / \mathrm{c} \\
\mathrm{w} / \mathrm{r}\end{array}$ & 0.28 & $\begin{array}{l}\mathrm{c} / \mathrm{c} \\
\mathrm{w} / \mathrm{r}\end{array}$ & 0.28 \\
\hline $\begin{array}{l}\Delta \mathrm{KДР}_{3-6} \\
\Delta \mathrm{FDS}_{3-6}\end{array}$ & 0.34 & $\begin{array}{l}\mathrm{c} / \mathrm{c} \\
\mathrm{w} / \mathrm{r}\end{array}$ & 0.27 & $\begin{array}{l}\mathrm{c} / \mathrm{c} \\
\mathrm{w} / \mathrm{r}\end{array}$ & 0.27 \\
\hline $\begin{array}{l}\triangle \mathrm{KДP}_{3-12} \\
\Delta \mathrm{FDS}_{3-12}\end{array}$ & 0.31 & $\begin{array}{l}\mathrm{c} / \mathrm{c} \\
\mathrm{w} / \mathrm{r}\end{array}$ & 0.34 & $\begin{array}{l}\mathrm{c} / \mathrm{c} \\
\mathrm{w} / \mathrm{r}\end{array}$ & 0.30 \\
\hline $\begin{array}{l}\Delta \mathrm{KCP}_{3-14} \\
\Delta \mathrm{FSS}_{3-14}\end{array}$ & 0.26 & $\begin{array}{l}\mathrm{c} / \mathrm{c} \\
\mathrm{w} / \mathrm{r}\end{array}$ & $\begin{array}{l}\mathrm{c} / \mathrm{c} \\
\mathrm{w} / \mathrm{r}\end{array}$ & $\begin{array}{l}\mathrm{c} / \mathrm{c} \\
\mathrm{w} / \mathrm{r}\end{array}$ & $\begin{array}{l}\mathrm{c} / \mathrm{c} \\
\mathrm{w} / \mathrm{r}\end{array}$ \\
\hline $\begin{array}{l}\Phi B_{14-\text { c cyт }} \\
\mathrm{EF}_{14 \text { th day }}\end{array}$ & $\begin{array}{l}\mathrm{c} / \mathrm{c} \\
\mathrm{w} / \mathrm{r}\end{array}$ & 0.26 & 0.32 & $\begin{array}{l}\mathrm{c} / \mathrm{c} \\
\mathrm{w} / \mathrm{r}\end{array}$ & $\begin{array}{l}\mathrm{c} / \mathrm{c} \\
\mathrm{w} / \mathrm{r}\end{array}$ \\
\hline $\begin{array}{l}\Phi B_{6-\text { й мec }} \\
\mathrm{EF}_{6 \text { th month }}\end{array}$ & 0.34 & 0.36 & 0.37 & 0.33 & 0.36 \\
\hline 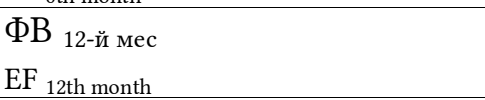 & 0.39 & 0.40 & 0.36 & 0.39 & 0.41 \\
\hline $\begin{array}{l}\Delta \Phi B_{3-14} \\
\Delta \mathrm{EF}_{3-14}\end{array}$ & $\begin{array}{l}\mathrm{c} / \mathrm{c} \\
\mathrm{w} / \mathrm{r}\end{array}$ & 0.27 & 0.39 & $\begin{array}{l}\mathrm{c} / \mathrm{c} \\
\mathrm{w} / \mathrm{r}\end{array}$ & $\begin{array}{l}\mathrm{c} / \mathrm{c} \\
\mathrm{w} / \mathrm{r}\end{array}$ \\
\hline $\begin{array}{l}\Delta \Phi B_{3-6} \\
\Delta \mathrm{EF}_{3-6}\end{array}$ & 0.38 & 0.35 & $\begin{array}{l}\mathrm{c} / \mathrm{c} \\
\mathrm{w} / \mathrm{r}\end{array}$ & 0.35 & 0.37 \\
\hline $\begin{array}{l}\Delta \Phi B_{3-12} \\
\Delta E_{3-12}\end{array}$ & 0.41 & 0.37 & 0.28 & 0.39 & 0.41 \\
\hline $\begin{array}{l}\Delta \Phi B_{14-6} \\
\Delta \mathrm{EF}_{14-6}\end{array}$ & 0.26 & $\begin{array}{l}\mathrm{c} / \mathrm{c} \\
\mathrm{w} / \mathrm{r}\end{array}$ & $\begin{array}{l}\mathrm{c} / \mathrm{c} \\
\mathrm{w} / \mathrm{r}\end{array}$ & $\begin{array}{l}\mathrm{c} / \mathrm{c} \\
\mathrm{w} / \mathrm{r}\end{array}$ & $\begin{array}{l}\mathrm{c} / \mathrm{c} \\
\mathrm{w} / \mathrm{r}\end{array}$ \\
\hline $\begin{array}{l}\Delta \Phi B_{14-12} \\
\Delta \mathrm{EF}_{14-12}\end{array}$ & 0.29 & $\begin{array}{l}\mathrm{c} / \mathrm{c} \\
\mathrm{w} / \mathrm{r}\end{array}$ & $\begin{array}{l}\mathrm{c} / \mathrm{c} \\
\mathrm{w} / \mathrm{r}\end{array}$ & 0.30 & 0.29 \\
\hline$\Delta \mathrm{IVCT}_{3-12}$ & 0.52 & $\begin{array}{l}\mathrm{c} / \mathrm{c} \\
\mathrm{w} / \mathrm{r}\end{array}$ & $\begin{array}{l}\mathrm{c} / \mathrm{c} \\
\mathrm{w} / \mathrm{r}\end{array}$ & $\begin{array}{l}\mathrm{c} / \mathrm{c} \\
\mathrm{w} / \mathrm{r}\end{array}$ & $\begin{array}{l}\mathrm{c} / \mathrm{c} \\
\mathrm{w} / \mathrm{r}\end{array}$ \\
\hline$\Delta \mathrm{E} / \mathrm{A}_{3-14}$ & $\begin{array}{l}\mathrm{c} / \mathrm{c} \\
\mathrm{w} / \mathrm{r}\end{array}$ & $\begin{array}{l}\mathrm{c} / \mathrm{c} \\
\mathrm{w} / \mathrm{r}\end{array}$ & $\begin{array}{l}\mathrm{c} / \mathrm{c} \\
\mathrm{w} / \mathrm{r}\end{array}$ & 0.55 & $\begin{array}{l}\mathrm{c} / \mathrm{c} \\
\mathrm{w} / \mathrm{r}\end{array}$ \\
\hline$\Delta \mathrm{E} / \mathrm{A}_{3-6}$ & $\begin{array}{l}\mathrm{c} / \mathrm{c} \\
\mathrm{w} / \mathrm{r}\end{array}$ & 0.48 & $\begin{array}{l}\mathrm{c} / \mathrm{c} \\
\mathrm{w} / \mathrm{r}\end{array}$ & 0.58 & $\begin{array}{l}\mathrm{c} / \mathrm{c} \\
\mathrm{w} / \mathrm{r}\end{array}$ \\
\hline $\begin{array}{l}\text { ИЛС } 6 \text { 6-й мec } \\
\text { ILMC }_{\text {6th month }}\end{array}$ & $\begin{array}{l}\mathrm{c} / \mathrm{c} \\
\mathrm{w} / \mathrm{r}\end{array}$ & $-0.29^{*}$ & $\begin{array}{l}\mathrm{c} / \mathrm{c} \\
\mathrm{w} / \mathrm{r}\end{array}$ & $\begin{array}{l}\mathrm{c} / \mathrm{c} \\
\mathrm{w} / \mathrm{r}\end{array}$ & $\begin{array}{l}\mathrm{c} / \mathrm{c} \\
\mathrm{w} / \mathrm{r}\end{array}$ \\
\hline $\begin{array}{l}\text { ИЛC }_{12-\text { й mec }} \\
\text { ILMC }_{12 \text { th month }}\end{array}$ & $\begin{array}{l}\mathrm{c} / \mathrm{c} \\
\mathrm{w} / \mathrm{r}\end{array}$ & $-0.31^{*}$ & $\begin{array}{l}\mathrm{c} / \mathrm{c} \\
\mathrm{w} / \mathrm{r}\end{array}$ & $\begin{array}{l}\mathrm{c} / \mathrm{c} \\
\mathrm{w} / \mathrm{r}\end{array}$ & $\begin{array}{l}\mathrm{c} / \mathrm{c} \\
\mathrm{w} / \mathrm{r}\end{array}$ \\
\hline \multicolumn{6}{|c|}{$\begin{array}{c}\text { 8-е сутки } \\
8^{\text {th }} \text { day }\end{array}$} \\
\hline $\begin{array}{l}\Delta \mathrm{KДР}_{3-12} \\
\Delta \mathrm{FDS}_{3-12}\end{array}$ & 0.27 & $\begin{array}{l}\mathrm{c} / \mathrm{c} \\
\mathrm{w} / \mathrm{r}\end{array}$ & $\begin{array}{l}\mathrm{c} / \mathrm{c} \\
\mathrm{w} / \mathrm{r}\end{array}$ & $\begin{array}{l}\mathrm{c} / \mathrm{c} \\
\mathrm{w} / \mathrm{r}\end{array}$ & $\begin{array}{l}\mathrm{c} / \mathrm{c} \\
\mathrm{w} / \mathrm{r}\end{array}$ \\
\hline $\begin{array}{l}\Phi B_{14-\mathrm{e} \mathrm{cyT}} \\
\mathrm{EF}_{14 \text { th dav }}\end{array}$ & $\begin{array}{l}\mathrm{c} / \mathrm{c} \\
\mathrm{w} / \mathrm{r}\end{array}$ & $\begin{array}{l}\mathrm{c} / \mathrm{c} \\
\mathrm{w} / \mathrm{r}\end{array}$ & 0.27 & $\begin{array}{l}\mathrm{c} / \mathrm{c} \\
\mathrm{w} / \mathrm{r}\end{array}$ & $\begin{array}{l}\mathrm{c} / \mathrm{c} \\
\mathrm{w} / \mathrm{r}\end{array}$ \\
\hline 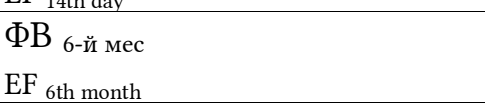 & 0.31 & $\begin{array}{l}\mathrm{c} / \mathrm{c} \\
\mathrm{w} / \mathrm{r}\end{array}$ & 0.30 & 0.28 & 0.29 \\
\hline 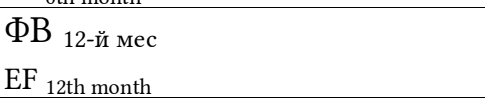 & 0.35 & 0.29 & 0.37 & 0.35 & 0.36 \\
\hline
\end{tabular}


Продолжение табл. 3

End of table 3

\begin{tabular}{|c|c|c|c|c|c|}
\hline \multirow{2}{*}{$\begin{array}{l}\text { Параметры допплер-Эхо-КГ } \\
\text { Parameters of doppler Echo-CG }\end{array}$} & \multicolumn{5}{|c|}{$\begin{array}{c}\text { Интегральные параметры } Ф С \\
\text { Integral parameters of the functional state }\end{array}$} \\
\hline & A & B & $\mathrm{C}$ & $\mathrm{D}$ & $\mathrm{H}$ \\
\hline \multicolumn{6}{|c|}{$\begin{array}{c}8 \text {-е сутки } \\
8^{\text {th }} \text { day } \\
\end{array}$} \\
\hline $\begin{array}{l}\Delta \Phi B_{3-14} \\
\Delta \mathrm{EF}_{3-14}\end{array}$ & 0.33 & 0.28 & 0.28 & 0.30 & 0.31 \\
\hline $\begin{array}{l}\Delta \Phi_{3-6} \\
\Delta E_{3-6}\end{array}$ & 0.39 & 0.33 & 0.27 & 0.32 & 0.34 \\
\hline $\begin{array}{l}\Delta \Phi_{3-12} \\
\Delta E_{3-12}\end{array}$ & 0.40 & 0.37 & 0.33 & 0.37 & 0.38 \\
\hline $\begin{array}{l}\Delta \Phi_{6-12} \\
\Delta \mathrm{EF}_{6-12}\end{array}$ & $\begin{array}{l}\mathrm{c} / \mathrm{c} \\
\mathrm{w} / \mathrm{r}\end{array}$ & $\begin{array}{l}\mathrm{c} / \mathrm{c} \\
\mathrm{w} / \mathrm{r}\end{array}$ & 0.25 & $\begin{array}{l}\mathrm{c} / \mathrm{c} \\
\mathrm{w} / \mathrm{r}\end{array}$ & $\begin{array}{l}\mathrm{c} / \mathrm{c} \\
\mathrm{w} / \mathrm{r}\end{array}$ \\
\hline$\Delta$ IVCT $_{3-12}$ & 0.70 & 0.53 & $\begin{array}{l}\mathrm{c} / \mathrm{c} \\
\mathrm{w} / \mathrm{r}\end{array}$ & $\begin{array}{l}\mathrm{c} / \mathrm{c} \\
\mathrm{w} / \mathrm{r}\end{array}$ & 0.57 \\
\hline$\Delta \mathrm{DT}_{3-14}$ & $\begin{array}{l}\mathrm{c} / \mathrm{c} \\
\mathrm{w} / \mathrm{r}\end{array}$ & 0.51 & $\begin{array}{l}\mathrm{c} / \mathrm{c} \\
\mathrm{w} / \mathrm{r}\end{array}$ & $\begin{array}{l}\mathrm{c} / \mathrm{c} \\
\mathrm{w} / \mathrm{r}\end{array}$ & 0.50 \\
\hline \multicolumn{6}{|c|}{$\begin{array}{l}\text { 14-е сутки } \\
14 \text { day }\end{array}$} \\
\hline 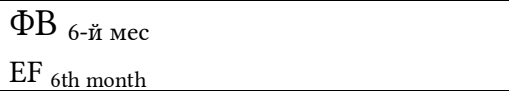 & 0.26 & 0.28 & 0.26 & $\begin{array}{l}\mathrm{c} / \mathrm{c} \\
\mathrm{w} / \mathrm{r}\end{array}$ & 0.26 \\
\hline 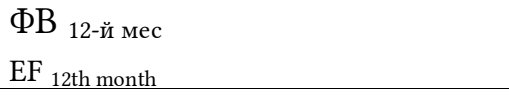 & 0.28 & 0.26 & 0.30 & 0.28 & 0.30 \\
\hline \multicolumn{6}{|c|}{$\begin{array}{c}\text { 6-й месяц } \\
6^{\text {th }} \text { month }\end{array}$} \\
\hline $\begin{array}{l}\Delta \Phi_{3-6} \\
\Delta E_{3-6}\end{array}$ & 0.32 & 0.32 & 0.28 & 0.27 & 0.29 \\
\hline $\begin{array}{l}\Delta \Phi B_{3-12} \\
\Delta E_{3-12}\end{array}$ & 0.33 & 0.33 & 0.28 & 0.29 & 0.29 \\
\hline $\begin{array}{l}\Delta \Phi B_{14-6} \\
\Delta \mathrm{EF}_{14-6}\end{array}$ & 0.28 & 0.28 & 0.28 & 0.26 & 0.26 \\
\hline $\begin{array}{l}\Delta \Phi B_{14-12} \\
\Delta \mathrm{EF}_{14-12}\end{array}$ & 0.30 & 0.30 & 0.28 & 0.28 & 0.27 \\
\hline \multicolumn{6}{|c|}{$\begin{array}{c}12 \text {-й месяц } \\
12^{\text {th }} \text { month }\end{array}$} \\
\hline $\begin{array}{l}\Delta \Phi_{3-12} \\
\Delta \mathrm{EF}_{3-12}\end{array}$ & 0.29 & 0.29 & 0.25 & $\begin{array}{l}\mathrm{c} / \mathrm{c} \\
\mathrm{w} / \mathrm{r}\end{array}$ & 0.27 \\
\hline $\begin{array}{l}\Delta \Phi B_{14-12} \\
\Delta \mathrm{EF}_{14-12}\end{array}$ & 0.25 & 0.25 & $\begin{array}{l}\mathrm{c} / \mathrm{c} \\
\mathrm{w} / \mathrm{r}\end{array}$ & $\begin{array}{l}\mathrm{c} / \mathrm{c} \\
\mathrm{w} / \mathrm{r}\end{array}$ & $\begin{array}{l}\mathrm{c} / \mathrm{c} \\
\mathrm{w} / \mathrm{r}\end{array}$ \\
\hline
\end{tabular}

Примечание: $\Delta$ - динамика показателя между этапами, указанными рядом; * - обратная корреляционная связь, с/с - слабая связь $(\mathrm{r}<0,25)$.

Note: $\Delta-$ the dynamics of the indicator between the stages indicated by a digit; ${ }^{*}$ - inverse correlation, w/r - weak relationship $(\mathrm{r}<0.25)$. FDS - final-diastolic size of the left ventricle, FSS - final-systolic size of the left ventricle, EF - ejection fraction, ILMC - index of local myocardial contractility.

миокарда на 6-й и 12-й мес и значением показателя В на 3 -и сут (0,29 и 0,31 соответственно).

Описанные результаты свидетельствуют о значимой взаимосвязи интегральных показателей ФС организма с динамикой структурных параметров миокарда, показателями систолодиастолической функции ЛЖ, характеристиками внутрисердечной гемодинамики. Особую значимость имели показатели А и В на первом этапе исследования (3-и сут), поскольку значение А связано с динамикой показателей КДР ЛЖ, КСР ЛЖ, ФВ, IVCT, а значение В - с динамикой величины ФВ и ИЛС миокарда.

Таким образом, использование современных математических методов анализа биоритмологических процессов в комплексной оценке ФС 
организма больных ОИМ позволило зарегистрировать снижение уровня адаптации сердечно-сосудистой системы, вегетативной и центральной регуляции, психоэмоционального состояния на всех этапах лечения и реабилитации больных ОИМ в течение года. Установлено, что в процессе госпитальных и постгоспитальных этапов реабилитации значения интегральных параметров A, C, D, Н возрастали, а величина уровня вегетативной регуляции снижалась. Доказано наличие значимой корреляционной связи между численными эквивалентами отдельных показателей $\Phi С$, допплер-Эхо-КГ и их динамикой на фоне реабилитации. Проведенное исследование обосновало целесообразность и перспективность использования комплексной оценки ФС организма больных ОИМ для характеристики течения кардиореабилитации. Полученные результаты позволяют предположить возможность использования параметров А и $\mathrm{B}$, регистрируемых на ранних этапах стационарного лечения, в качестве прогностических критериев эффективности восстановления внутрисердечной гемодинамики у больных ОИМ в госпитальный и постгоспитальный периоды реабилитации. Результаты работы свидетельствуют о необходимости дальнейших исследований в этой области, направленных на оценку эффективности лечебно-профилактических мероприятий у больных ОИМ.

\section{КОНФЛИКТ ИНТЕРЕСОВ}

Авторы декларируют отсутствие явных и потенциальных конфликтов интересов, связанных с публикацией настоящей статьи.

\section{СООТВЕТСТВИЕ ПРИНЦИПАМ ЭТИКИ}

Протокол исследования одобрен региональным этическим комитетом при ФГБОУ ВО «Курский государственный медицинский университет» Минздрава России (протокол № 9 от 09.11.15 г.). Каждым пациентом было подписано индивидуальное согласие на участие в исследовании.

\section{ИСТОЧНИКИ ФИНАНСИРОВАНИЯ}

Авторы заявляют об отсутствии финансирования.

\section{ЛИЧНЫЙ ВКЛАД АВТОРОВ}

Михин В.П. - научный руководитель исследования, Коробова В.Н. - разработка концепции и дизайна исследования, анализ и интерпретация полученных данных, Харченко А.В. - проведение допплерЭхо-КГ, Громнацкий Н.И. - проверка критически важного интеллектуального содержания, Выгодин В.А. - статистическая обработка данных.

\section{ЛИТЕРАTУРA/REFERENCES}

1. Алехин М.Н. Проблемы и перспективы эхокардиографической оценки диастолической функции левого желудочка сердца. Кардиология. 2017;57(1): 71-75. DOI: 10.18565/cardio.2017.1.71-75 [Alekhin M.N. Problems and Perspectives of Echocardiographic Evaluation of the Left Ventricular Diastolic Function. Kardiologiya. 2017;57(1): 71-75 (in Russ.)]

2. Алехин М.Н., Гришин А.М., Петрова О.А. Эхокардиографическая оценка диастолической функции левого желудочка сердца у пациентов с сохранной фракцией выброса. Кардиология. 2017;57(2): 40-45. DOI: 10.18565/cardio.2017.2.40-45 [Alekhin M.N., Grishin A.M., Petrova O.A. The Evaluation of Left Ventricular Diastolic Function by Echocardiography in Patients With Preserved Ejection Fraction. Kardiologiya. 2017; 57(2): 40-45 (in Russ.)]

3. Аронов Д.М., Иоселиани Д.Г., Бубнова М.Г., Красницкий В.Б., Гринштейн Ю.И., Гуляева С.Ф., Ефремушкин Г.Г., Лямина Н.П. Результаты российского рандомизированного контролируемого клинического исследования по оценке клинической эффективности комплексной годичной программы реабилитации с включением физических тренировок у трудоспособных больных, перенесших острый инфаркт миокарда на фоне артериальной гипертонии. Вестник восстановительной медицины. 2017; 5(81): 2-11. [Aronov D.M., Ioseliani D.G., Bubnova M.G., Krasnitsky V.B., Grinshtein Y.I., Gulyaeva S.F., Efremushkin G.G., Lyamina N.P. Results of a russian randomized controlled clinical trial evaluating the clinical effectiveness of a comprehensive annual rehabilitation program with the inclusion of physical training in ablebodied patients who underwent acute myocardial i nfarction in the presence of arterial hypertension. Vestnik vosstanovitel'noy meditsiny. 2017; 5(81): 2-11 (in Russ.)]

4. Бокерия Л.А., Алехин М.Н., Машина Т.В., Мрикаев Д.В., Голухова Е.З. Современные ультразвуковые технологии в кардиологии и кардиохирургии: монография. Москва: НМИЦ ССХ им. А.Н. Бакулева, 2018. 140 с. [Bokeriya L.A., Alekhin M.N., Mashina T.V., Mrikayev D.V., Golukhova E.Z Modern ultrasound technologies in cardiology and cardiac surgery: a monograph. Moscow: Bakulev Scientific Center of Cardiovascular Surgery, 2018. 140 p. (in Russ.)]

5. Грузин Н.В., Смирнов К.Ю., Трофимов Е.В., авторы, Грузин Н.В., К.Ю. Смирнов, Е.В. Трофимов, патентообладатели. Способ контроля функционального состояния биологического объекта. RU 219153927 октября 2002 г. [Gruzin N.V., Smirnov K.Yu., E.V. Trofimov E.V., authors, Gruzin N.V., Smirnov K.Yu., Trofimov E.V., assignees. The method of controlling the functional state of a biological object. Russian Federation patent 2191539 2002 Oct 27 (in Russ.)]

6. Михин В.П., Коробова В.Н., Харченко А.В., Громнацкий Н.И., Чернятина М.А., Спасский А.А., Ка- 
юшников А.Б., Ледовский С.М., Маркина Т.Н. Особенности функционального состояния больных острой коронарной патологией и влияние на них уровня физической реабилитации на момент выписки. Научные ведомости БелГУ. Серия: Медицина. Фармация. 2018; 41(3): 400-409. DOI: 10.18413/2075-4728-2018-41-3-400-409 [Mikhin V.P., Korobova V.N., Kharchenko A.V., Chernjatina M.A., Gromnackiy N.I., Spasskiy A.A., Kayushnikov A.B., Ledovskiy S.M., Markina T.N. Features of the functional state of patients with acute coronary pathology and the influence on them of the level of physical rehabilitation at the discharge moment. Nauchnyye vedomosti BelGU. Seriya: Meditsina. Farmatsiya. 2018; 41(3): 400-409 (in Russ.)]

7. Пименов Ю.С., Юрикова М.В., Данилова В.Н. Исследование терапевтической эффективности эфирных масел в коррекции психоэмоционального напряжения у больных с хронической болью в нижней части спины. Вестник медицинского института "РЕАВИЗ": реабилитащия, врач и здоровье. 2013;3: 27-30 [Pimenov Yu.S., Yurikova M.V., Danilova V.N. Issledovaniye terapevticheskoy effektivnosti efirnykh masel $\mathrm{v}$ korrektsii psikhoemotsional'nogo napryazheniya u bol'nykh s khronicheskoy bol'yu v nizhney chasti spiny. Vestnik meditsinskogo instituta "REAVIZ": reabilitatsiya, vrach i zdorov'ye. 2013; 3: 27-30 (in Russ.)]

8. Пономаренко И.В., Сукманова И.А. Традиционные факторы риска и генные мутации тромбоза, ассоциированные с острым коронарным синдромом у пациентов молодого возраста. Кардиология. 2019; 59(1S): 19-24. DOI: 10.18087/cardio.2602 [Ponomarenko I.V., Sukmanova I.A. Thrombosis risk factors and gene mutations in young age patients with acute coronary syndrome. Kardiologiya. 2019;59(1S): 19-24 (in Russ.)]

9. Савченко Е.С., Назаров Р.Н., Апчел А.В., Патрушев А.В., Павлова Н.В. Коррекция психовегетативных расстройств в процессе комплексного лечения больных хроническими дерматозами. Вестник Российской военно-медицинской академии. 2012; 3(39): 107-111 [Savchenko E.S., Nazarov R.N., Patrushev A.V., Apchel A.V., Pavlova N.V. Correction of psychoautonomic disorders in the course of complex treatment of patients with chronic dermatoses. Vestnik Rossiyskoy voyenno-meditsinskoy akademii. 2012; 3(39): 107-111 (in Russ.)]

10. Сараев И.А., Васильева Д.А. Диагностическая значимость организации системного гомеокинеза при остром коронарном синдроме. Современные проблемы науки и образования. 2018; 1: 59 [Saraev I.A., Vasileva D.A. Diagnostic significance of organization of systemic gomeokinesis in acute coronary syndrome. Sovremennyye problemy nauki i obrazovaniya. 2018; 1: 59 (in Russ.)]

11. Смирнов Ю.А., Смирнов К.Ю., Жаров М.В., Свистуленко В.А., авторы, Смирнов Ю.А., Смирнов К.Ю., Жаров М.В., Свистуленко В.А., патентообладатели. Динамическое наблюдение и прогноз изменения функционального состояния пациента: свидетельство о регистрации программы для ЭВМ RU 2003611173. 20 мая 2003 г. [Smirnov Yu.A.,
Smirnov K.Yu., Zharov M.V., Svistulenko V.A., inventors, Smirnov Yu.A., Smirnov K. Yu., Zharov M.V., Svistulenko V.A., assignees. Dynamic observation and prediction of a change in the functional state of a patient: certificate of registration of a computer program. Russian Federation patent RU 20036111732003 May 20 (in Russ.)]

12. Смирнов К.Ю., Смирнов Ю.А. Разработка и исследование методов математического моделирования и анализа биоэлектрических сигналов. СанктПетербург: издательство неизвестно, 2001. 43 с. [Smirnov K.Yu., Smirnov Yu.A. Development and research of methods for mathematical modeling and analysis of bioelectric signals. Saint-Petersburg: publisher unknown, 2001. 43 p. (in Russ.)]

13. Чудакова Е.А., Галяутдинов Г.С. Диастолическая дисфункция миокарда, особенности ремоделирования сердца у больных с острым коронарным синдромом и постинфарктным кардиосклерозом. Практическая медицина. 2011; 4(52): 93-96 [Chudakova E.A., Galyautdinov G.S. Diastolic myocardial dysfunction, remodeling the heart in patients with acute coronary syndromes and postinfarction cardiosclerosis. Prakticheskaya meditsina. 2011; 4(52): 93-96 (in Russ.)]

14. Agarwal S., Sud K., Takkar B., Menon V., Jaber W.A., Kapadia S.R. Changing Trends of Atherosclerotic Risk Factors Among Patients with Acute Myocardial Infarction and Acute Ischemic Stroke. Am $\mathcal{F}$ Cardiol. 2017; 119(10): 1532-1541. DOI: 10.1016/j.amjcard.2017.02.027.

15. Ibanez B., James S., Agewall S., Antunes M.J., Bucciarelli-Ducci Ch., Bueno H., Caforio A.L.P., Crea F., Goudevenos J.A., Halvorsen S., Hindricks G., Kastrati A., Mattie J., Lenzen M.J., Prescott E., Roffi M., Valgimigli M., Varenhorst Ch., Vranckx P., Widimsky P. ESC Guidelines for the management of acute myocardial infarction in patients presenting with ST-segment elevation. Rev Esp Cardiol (Engl Ed). 2017; 70(12): 1082. DOI: 10.1016/j.rec.2017.11.010.

16. Roffi M., Andreotti F., Bax J.J., Borger M.A., Brotons C., Chew D.P., Gencer B., Hasenfuss G., Kjeldsen K., Lancellotti P., Landmesser U., Mehilli J., Mukherjee D., Storey R.F., Windecker S., Collet J.P., Mueller C., Valgimigli M., Zamorano J.L., Badimon L., Barón-Esquivias G., Bueno H., Aboyans V., Dean V., Achenbach S., Baumgartner H., Kirchhof P., Agewall S., Carerj S., Piepoli M.F., Erol Ç., Fitzsimons D., Lip G.Y.H., Nihoyannopoulos P., Kolh P., Ponikowski P., Torbicki A., Gaemperli O., Carneiro A.V., Chilingaryan A., Weidinger F., Najafov R., Sinnaeve P.R., Terzić I., Postadzhiyan A., Miličić D., Eftychiou C., Widimsky P., Bang L., El Etriby A., Marandi T., Pietilä M., Kedev S., Koning R., Aladashvili A., Neumann F.J., Tsioufis K., Becker D., Guðnason T., Matetzky S., Bolognese L., Mussagaliyeva A., Beishenkulov M., Latkovskis G., Serpytis P., Pereira B., Magri C.J., Grosu A., Abir-Khalil S., Larsen A.I., Budaj A., Mimoso J.M.V., Ginghina C., Averkov O., Nedeljkovic M.A., Studenčan M., Barrabés J.A., Held C., Rickli H., Peters R.J.G., Mourali M.S., Atalar E., Swanson N., Parkhomenko A., Halle M., Baigent C., Bugiardini R., Casselman F., Cuisset T., 
Patrono C. Рабочая группа европейского кардиологического общества (ESC). Рекомендации ESC по ведению пациентов с острым коронарным синдромом без стойкого подъема сегмента ST 2015. Российский кардиологический журнал. 2016; 21(3): 9-632016; 21(3): 9-63 [Roffi M., Andreotti F., Bax J.J., Borger M.A., Brotons C., Chew D.P., Gencer B., Hasenfuss G., Kjeldsen K., Lancellotti P., Landmesser U., Mehilli J., Mukherjee D., Storey R.F., Windecker S., Collet J.P., Mueller C., Valgimigli M., Zamorano J.L., Badimon L., Barón-Esquivias G., Bueno H., Aboyans V., Dean V., Achenbach S., Baumgartner H., Kirchhof P., Agewall S., Carerj S., Piepoli M.F., Erol Ç., Fitzsimons D., Lip G.Y.H., NiIhoyannopoulos P., Kolh P., Ponikowski P., Torbicki A., Gaemperli O., Carneiro A.V., Chilingaryan A., Weidinger F., Najafov R., Sinnaeve P.R., Terzić I., Postadzhiyan A., Miličić D., Eftychiou C., Widimsky P., Bang L., El Etriby A., Marandi T., Pietilä M., Kedev S., Koning R., Aladashvili A., Neu- mann F.J., Tsioufis K., Becker D., Guðnason T., Matetzky S., Bolognese L., Mussagaliyeva A., Beishenkulov M., Latkovskis G., Serpytis P., Pereira B., Magri C.J., Grosu A., Abir-Khalil S., Larsen A.I., Budaj A., Mimoso J.M.V., Ginghina C., Averkov O., Nedeljkovic M.A., Studenčan M., Barrabés J.A., Held C., Rickli H., Peters R.J.G., Mourali M.S., Atalar E., Swanson N., Parkhomenko A., Halle M., Baigent C., Bugiardini R., Casselman F., Cuisset T., Patrono C. European society of cardiology (ESC) working group. ESC guidelines for the management of patients with acute coronary syndrome without persistent ST-segment elevation 2015. Russian Journal of Cardiology. 2016; 21(3): 9-63 (in Russ.)]

17. Shah N., Kelly A-M., Cox N., Wong C., Soon K. Myocardial Infarction in the "Young": Risk Factors, Presentation, Management and Prognosis. Heart Lung Circ. 2016; 25(10):955-960. DOI: 10.1016/j.hlc.2016.04.015

Поступила в редакцию 09.01.2019 Подписана в печать 21.03.2019

Для цитирования: Михин В.П., Коробова В.Н., Харченко А.В., Громнацкий Н.И., Выгодин В.А. Взаимосвязь изменений показателей функционального состояния и допплер-эхокардиографии у больных острым инфарктом миокарда на фоне реабилитации. Курский научно-практический вестник «Человек и его здоровье». 2019;(1):41-50. DOI: 10.21626/vestnik/2019-1/05.

\title{
CORRELATION OF CHANGES IN THE INDICATORS OF FUNCTIONAL STATE AND DOPPLER-ECHOCARDIOGRAPHY IN PATIENTS WITH ACUTE MYOCARDIAL INFARCTION AGAINST THE BACKGROUND OF REHABILITATION
}

\author{
○ Mikhin V.P. ${ }^{1}$, Korobova V.N. ${ }^{1}$, Kharchenko A.V. ${ }^{1}$, Gromnatsky N.I. ${ }^{1}$, Vygodin V.A. ${ }^{2}$ \\ ${ }^{1}$ Kursk State Medical University (KSMU) \\ 3, K. Marx st., Kursk, Kursk region, 305041, Russian Federation \\ ${ }^{1}$ National Medical Research Center for Preventive Medicine (NMRCPM) \\ 10, Petroverigskiy lane, Moscow, 101990, Russian Federation
}

\begin{abstract}
Objective. The purpose of the study was to assess the integral indicators of the functional state (FS) of the body, the parameters of central and intracardiac hemodynamics in patients with acute myocardial infarction in the process of hospital and post-hospital rehabilitation, and to study the relationships between the received data.

Materials and methods. An open, randomized study included 111 people (76 men and 35 women) aged 40 to 65 years (57.2 \pm 0.6$)$. The study of the body FS was carried out using the Dynamic-Omega-M hardware-software complex on the 3rd, 8th, and 14th days and by the 6th and 12th months. Doppler echocardiography was performed on the 3rd and 14th days of treatment, 6 and 12 months after discharge.

Results. The obtained results allowed to establish a decrease in the values of integral indices of FS at the stages of hospital and post-hospital rehabilitation, the presence of myocardial hypertrophy, disorders of diastolic function of the heart and local contractility. Against the background of rehabilitation, positive dynamics of FS parameters and indices characterizing intracardiac hemodynamics was established. Significant correlations were established between the values of FS parameters and echocardiographic parameters and their dynamics during the study.

Conclusion. The research justified the expediency of using the integrated parameters of FS for a comprehensive assessment of the status of patients with acute myocardial infarction and as prognostic criteria for the effectiveness of reha-

Keywords: acute myocardial infarction; functional state; intracardiac hemodynamics; central hemodynamics; rehabili-
\end{abstract} bilitation. tation.

Mikhin Vadim P. - DM, Professor, Head of Department of Internal Diseases No 2, KSMU, Kursk, Russian Federation. ORCID iD: 0000-0002-5398-9727. E-mail: mikhinvp@yandex.ru

Korobova Viktoriya N. - Assistant of Pathophysiology Department, KSMU, Kursk, Russian Federation. ORCID iD: 0000-0002-27373435. E-mail: viktoria.korobova@mail.ru (correspondence author) 
Kharchenko Aleksandr V. - PhD in Medicine, Associate Professor of Department of Internal Diseases No 2, KSMU, Kursk, Russian Federation. ORCID iD: 0000-0001-8636-3096. E-mail: hav46@mail.ru

Gromnatsky Nikolay I. - DM, Professor, Professor of Department of Internal Diseases No 2, KSMU, Kursk, Russian Federation. E-mail: gromnackini@yandex.ru

Vygodin Vladimir A. - Senior Researcher of Laboratory of Medical Biostatistics, NMRCPM, Moscow, Russian Federation. E-mail: vladimirvygodin@mail.ru

\section{CONFLICT OF INTEREST}

The authors declare the absence of obvious and potential conflicts of interest related to the publication of this article.

\section{SOURCE OF FINANCING}

The authors state that there is no funding for the study.

\section{CONFORMITY WITH THE PRINCIPLES OF ETHICS}

The study protocol was approved by the Ethical Committee under Kursk state medical university (Protocol No. 9 of
09.11.2015). Each patient signed the individual consent to participate in the study.

\section{AUTHORS CONTRIBUTION}

Mikhin V.P. - scientific research guidance, Korobova V.N. - developing the research concept and design, data analyzing and interpreting the results, Kharchenko A.V. - conducting Doppler echocardiography, Gromnatsky N.I. - critical revision of the manuscript for important intellectual content, Vygodin V.A. - statistical data analysis.

Received 09.01.2019

Accepted 21.03.2019

For citation: Mikhin V.P., Korobova V.N., Kharchenko A.V., Gromnatsky N.I., Vygodin V.A. Correlation of changes in the indicators of functional state and Doppler-echocardiography in patients with acute myocardial infarction against the background of rehabilitation. Kurskiy nauchno-prakticheskiy vestnik "Chelovek i ego zdorov'ye" = Kursk Scientific and Practical Bulletin "Man and His Health". 2019;(1): 41-50. DOI: 10.21626/vestnik/2019-1/05. 